\title{
Article \\ Origin and Justification of the Use of the Arrhenius Relation to Represent the Reaction Rate of the Thermal Decomposition of a Solid
}

\author{
Benjamin Batiot $^{1, *}$, Thomas Rogaume ${ }^{1}\left(\mathbb{D}\right.$, Franck Richard $^{1}$, Jocelyn Luche ${ }^{1} \mathbb{D}$, Anthony Collin ${ }^{2}(\mathbb{D}$, \\ Eric Guillaume ${ }^{3}$ and José Luis Torero ${ }^{4}$
}

\section{check for} updates

Citation: Batiot, B.; Rogaume, T; Richard, F.; Luche, J.; Collin, A.; Guillaume, E.; Torero, J.L. Origin and Justification of the Use of the Arrhenius Relation to Represent the Reaction Rate of the Thermal Decomposition of a Solid. Appl. Sci. 2021, 11, 4075. https://doi.org/ 10.3390/app11094075

Academic Editor:

Antonella D'Alessandro

Received: 30 March 2021

Accepted: 27 April 2021

Published: 29 April 2021

Publisher's Note: MDPI stays neutral with regard to jurisdictional claims in published maps and institutional affiliations.

Copyright: (c) 2021 by the authors. Licensee MDPI, Basel, Switzerland. This article is an open access article distributed under the terms and conditions of the Creative Commons Attribution (CC BY) license (https:// creativecommons.org/licenses/by/ $4.0 /)$.
1 Département Fluides, Thermique, Combustion, Institut $\mathrm{P}^{\prime}$, UPR 3346 CNRS, Université de Poitiers, ISAE-ENSMA, CEDEX 9, 86073 Poitiers, France; thomas.rogaume@univ-poitiers.fr (T.R.); franck.richard@univ-poitiers.fr (F.R.); jocelyn.luche@ensma.fr (J.L.)

2 CNRS, LEMTA, Université de Lorraine, 54000 Nancy, France; anthony.collin@univ-lorraine.fr

3 Efectis France, Espace Technologique, Route 1'Orme des Merisiers, 91193 Saint Aubin, France; eric.guillaume@efectis.com

4 Department of Civil, Environmental and Geomatic Engineering, University College London, London WC1E 6DE, UK; j.torero@ucl.ac.uk

* Correspondence: benjamin.batiot@univ-poitiers.fr; Tel.: +33-5-49-24-94-88

\begin{abstract}
Degradation models are commonly used to describe the generation of combustible gases when predicting fire behavior. A model may include many sub-models, such as heat and mass transfer models, pyrolysis models or mechanical models. The pyrolysis sub-models require the definition of a decomposition mechanism and the associated reaction rates. Arrhenius-type equations are commonly used to quantify the reaction rates. Arrhenius-type equations allow the representation of chemical decomposition as a function of temperature. This representation of the reaction rate originated from the study of gas-phase reactions, but it has been extrapolated to liquid and solid decomposition. Its extension to solid degradation needs to be justified because using an Arrheniustype formulation implies important simplifications that are potentially questionable. This study describes these simplifications and their potential consequences when it comes to the quantification of solid-phase reaction rates. Furthermore, a critical analysis of the existing thermal degradation models is presented to evaluate the implications of using an Arrhenius-type equation to quantify mass-loss rates and gaseous fuel production for fire predictions.
\end{abstract}

Keywords: Arrhenius; solid kinetics; thermal degradation; modeling

\section{Introduction}

Uncontrolled fires pose a significant threat to life and property. Predictions of the evolution of fire are essential for risk assessment and consequence mitigation. However, fires are controlled by complex chemical and physical processes (fluid mechanics, heat transfer, chemical kinetics, solid decomposition, etc.), making detailed predictions difficult, time-consuming and often inaccurate. Furthermore, uncertainty assessment is complex because uncertainties can come from many directions that range from model parameters to the structure and assumptions embedded in the models [1]. Thermal decomposition of solid materials is one of the processes that are complex to model and carries significant uncertainties.

All aspects of fire growth modeling require some representation of the thermal decomposition of condensed combustible materials. Earlier models relied on simple empirical laws that involved major simplifications and that were mainly based on heat transfer [2,3]. A perfect example is the earlier treatment of ignition, where the inert heating approach was used towards establishing ignition criteria. Reviews of these simple ignition models can be found in classic textbooks $[2,3]$. 
Among the most simplified representations of the onset of combustion are concepts, such as ignition temperatures. By defining such a criterion, the treatment of thermal decomposition is ultimately avoided, and ignition is defined purely as a thermal process [4]. An empirical determination of an ignition temperature (i.e., the surface temperature at ignition) allows the calculation of parameters, such as the time to ignition, or serves as inputs for simple flame spread models [2,3]. More detailed formulations have avoided using an ignition temperature and characterized ignition using a critical mass-loss rate [2]. Once again, the mass-loss rate is expressed in thermal terms, and these models still do not consider a detailed analysis of thermal decomposition. An empirically determined critical mass flux for ignition serves as the ignition criterion. This ignition criterion has the advantage of using a quantity that can be measured effectively but also provides a direct link with physical concepts, such as flammability limits that an ignition temperature cannot deliver [3].

The introduction of high-performance computing has made these simplifications unnecessary resulting in the proliferation of many thermal decomposition models that serve as inputs to fire dynamics models [5-9]. The objective of these models goes beyond establishing ignition criteria and enables calculating the production of fuel during all stages of a fire. The production of fuel is a key parameter that serves as an input for any combustion model.

Many computational tools have been developed, and each tool has its own specific approach to the modeling of these terms (FireFoam [5], GPyro [6], ThermaKin [7] or Pyropolis [8]), but a generic representation of the conservation law for fuel is represented by Equation (1):

$$
\frac{\partial}{\partial t}\left(\frac{\rho_{s}}{\rho_{s}(0)}\right)=\sum_{i=1}^{m}\left(\sum_{\gamma \in P_{i}} v_{\gamma} r_{\gamma}-\sum_{\epsilon \in R_{i}} r_{\epsilon}\right)
$$

In this equation $\rho_{s}$ represents the mass volume of the solid, $\rho_{s}(0)$ the initial mass volume of the solid, $v_{\gamma}$ the stœchiometric coefficient, $r_{\gamma}$ is the production rate, $r_{\epsilon}$ the consumption rate and $m$ the number of species involved during the degradation process. $P_{i}$ represents the reactions involving the production of the specie $i$ and $R_{i}$ represents those, which use the species $i$ as a reactant. The key to solving Equation (1) is to determine the production and consumption rates.

The first studies are attempting to determine the production and consumption rates, i.e., chemical kinetics, date to the 19th century [10]. Empirical formulations first emerged, and then they were followed by many authors attempting to use statistical physics theory to justify the form of the empirical laws previously found [11]. These studies drew a parallel between gas phase and solid phase kinetics. Dating to the middle of the 20th-century, several authors questioned the form of the statistical distributions when applied to the reactions resulting in the thermal degradation of solids [12,13].

Among the different approaches, the most common one establishes that for a nonoxidative degradation reaction, the production rate $(r)$ is given by:

$$
r=\left(\frac{\rho_{s}}{\rho_{s}(0)}\right)^{n_{s}} A^{p f} e^{-\frac{E_{a c t}}{R T}}
$$

where $n_{s}$ represents the reaction order, $A^{p f}$ the pre-exponential factor, $E_{a c t}$ the activation energy, $R$ the perfect gas constant and $T$ the temperature. Then, Equation (2) is composed of the quantity of reactive mass $\left(\left(\frac{\rho_{s}}{\rho_{s}(0)}\right)^{n_{s}}\right)$ and the reaction rate relation $\left(A^{p f} e^{-\frac{E_{a c t}}{R T}}\right)$. This last relation is known as the Arrhenius relation, first established in 1889 by Arrhenius [10].

All the current models [5-8] have their own representations of the transport equations consistent with conventional conservation equations [4]. While each author prioritizes specific phenomena relevant to the processes they intend to model, they adopt different simplifications and choose different expressions to model individual phenomena. The same relation (Arrhenius form) is used in all solid degradation models [13-15]. 
Most studies concerned with thermal degradation of solids have not been devoted to formulation itself, but to better understand the input parameters (pre-exponential or the activation energy), to find optimized methodologies to extract these parameters from data, to establish the mechanisms by which fuel is produced and transported ( $n$th order, diffusion ... ) and to describe the influence of temperature on the different parameters [15]. A detailed example of such studies is reference [16], where it is clearly established that the form of the reaction rate expressions needs to be significantly modified when applied to different materials, many of relevant to fire safety.

It is, therefore, clear that there is great confidence in the capacity of the Arrhenius representation to describe the thermal degradation of solids. Despite this confidence, there is almost no work that serves to unequivocally establish the theoretical underpinnings of the Arrhenius relation. The work of Galwey and Brown [17] is one the most extensive study devoted to the theory underpinning the Arrhenius relation. While the authors conclude that such a model is inapplicable to the immobilized constituents of a solid, they also indicate that the energy distribution functions at the interface (where reactions occur) are analogous to those in the Maxwell-Boltzmann distribution functions used to justify using Arrhenius for gas-phase reactions. While important, this study focuses on degradation occurring as a single step in an infinitesimally thin interface and, therefore, is not conclusive as support to the wide range of applications of the Arrhenius relation in fire modeling.

Consequently, this article is focused on the Arrhenius relation itself. The objective is to review the basis for the reaction rate relation used for solid degradation and to provide any available justification for selecting any specific form of this formulation. This paper is not intended to prove or disproof the validity of such a relation. Instead, it is directed to organize existing evidence so that those using these expressions in fire modeling can indicate the level of confidence behind the expression.

\section{Background of the Arrhenius law}

The basic models that represented the reaction rate appeared at the end of the nineteenth century. The modern approach to chemical kinetics due to Wilhelmy, who in 1850 first formulated a kinetic law that described the first-order reaction (see $[11,18]$ ). This formulation gives a proportionality between the rate of the process and the amount of matter remaining during the sugar cane reaction [18]. Thereafter, Guldberg and Waage, between 1864 and 1879 [11], developed the mathematical reaction kinetic expression that was later formalized by Van't Hoff in 1884 [19].

After Van't Hoff, Arrhenius introduced the temperature dependence of the reaction rate and introduced the concept of activation energy in $1889[10,20]$. These concepts were then applied to the solid reactions by using the concept of single-step reaction and the introduction of the conversion function (Lewis in 1905 [21]). After Lewis, the multistep nature of solid-state reactions became increasingly obvious and progressively different complexities have been added to better represent different observed forms of degradation. For more details and explanation of the different empirical approaches and interpretations, the reader can consult references $[11,18]$.

However, these approaches are empirical, and none of them brings a theoretical analysis or justification of the Arrhenius relation itself. Although the Arrhenius relation provides good agreement with experimental data, it is not a theoretical justification for the form of the equation.

The next section aims to build from the literature in several domains of physics and chemistry the available information that could serve to provide a theoretical justification for using this kinetic equation in the modeling of solid degradation.

\section{Theoretical Approach}

The theoretical foundations associated with complex kinetic decomposition models are defined at length scales corresponding to atoms or molecules $\left(\approx 10^{-11}-10^{-9} \mathrm{~m}\right)$ and typically span time scales defined by atomic motion $\left(\approx 10^{-12}\right.$ s or less [22]). Therefore, the 
data that is used to support these theoretical foundations must be collected at these time and length scales [23]. These data are expected to respond to questions required for the assessment of numerous thermodynamic variables [24].

Many of these variables cannot be resolved when addressing problems that involve larger scales, as will be typical of all fire problems and combustible materials. However, statistical mechanics principles allow the determination of the probability of the occurrence of any particular state in the system. Consequently, if the prediction of the exact behavior of a single molecule is impossible, using statistical methods allows finding the average behavior of an ensemble consisting of many molecules [25]. Then, statistical averaging is used to increase the scale and reduce the number of variables without losing the necessary molecular-level information. Moreover, finally, this theory is applied to kinetics.

Kinetic laws provide macroscopic representations of the thermal degradation of materials. Nevertheless, they represent phenomena occurring at the atomic level. Statistical thermodynamics provides the tools to bridge the gap between these scales. Therefore, this work begins from the smallest scale and explores the assumptions embedded in the statistical methods used to achieve the macroscopic representation.

\subsection{Statistical Mechanics to Describe Energy}

At the origin of every transformation, there is energy. The energy stored in any particle (atoms or molecules) can be analyzed using Schrödinger's equation [26] and used as a vehicle to provide a physical basis for the upscaling process. The resolution of the Schrödinger's equation [25] gives us information about the quantum particles and their state of energy. Thus, the energy of a system is composed of the kinetic energy of each part of the system and the potential energy of all the interactions between all parts of the system.

For a perfect monoatomic gas, the interactions can be considered negligible. Hence the global energy can be described as the sum of the energy of all parts. If the interactions cannot be neglected, then the forces between atoms need to be included in the description of the overall energy of the system. The potential energy of the interactions adds a significant level of complexity. Indeed, in many cases, the interactions are not well-known. If strong interactions exist, then assuming that the global energy of the system is equal to the sum of the energy of all elementary particles (assumption of equipartition) brings uncertainty and potentially significant errors about using the Boltzmann statistics in the Arrhenius equation development. This is an initial and first key assumption that has yet to be demonstrated for materials of the complexity of those involved in fires.

This is a strong assumption, but it enables the description of the energy repartition by the following equation:

$$
U=\sum_{i=0}^{I} E_{i} n_{i}
$$

Here $U$ represents the total energy of the system, $E_{i}$ the level of energy in the state $i$ and $n_{i}$ the number of particles in the energetic state $i$ (this definition is consistent with the quantum mechanics definitions, see [27]). The total number of particles $N$ in all energetic states $i$ is given by:

$$
N=\sum_{i} n_{i}
$$

Based on the previous assumptions, Boltzmann uses statistical mechanics $[25,28]$ to develop the path between the microscopic and macroscopic scale (see Appendix A about the Boltzmann development and the key steps with the Equation (A4) and the Equation (A15), both use the Equations (3) and (4)). To do that, Boltzmann included the configurations and weights of all molecules and all molecule states. Indeed, at any instance, there will be $n_{0}$ molecules in the state 0 with energy $E_{0}$ and so forth for states 1,2 , etc. (see Appendix A, Equation (A1)).

To account for the micro-state, in Boltzmann's statistics, the particles must be identic and distinguishable [29], here it is mean that the position and the velocity of each particle are known (contrary to quantum particles, for example). This is the second key assumption. 
Finally, after an extensive derivation (see Appendix A), Boltzmann determines the following equation:

$$
\frac{n_{i}}{N}=\frac{g_{i}}{Z} e^{-\frac{E_{i}}{k_{b} T}}
$$

Thus, Equation (5) represents the Boltzmann distribution, which describes a number $n_{i}$ of particles in the energetic state $E_{i}$. In this equation, $g_{i}$ represents the degeneracy of the state $i, Z$ is the partition function (see Equation (A12) in Appendix A), $k_{b}$ is the Boltzmann constant and $T$ the temperature [29].

Equation (5) describes the behavior of an ensemble of particles by a macroscopic expression, and thus the transition between microscopic and macroscopic scale is mathematically formalized.

The Boltzmann equation and its development is the key concept that enables the understanding of the kinetic equation and any potential limitations to it. In the following sections, the main assumptions used in developing Equation (5) are further analyzed to identify other key issues of relevance. These assumptions are then evaluated in the context of the general kinetic law. In doing so, this work extends the study by Galwey and Brown [17].

\subsection{The Formulation of the Arrhenius Form}

When considering a reaction at equilibrium in which a reactant $A$ is transformed to a product $B$ (reactants and products can be solids, liquids or gaseous). $A$ and $B$ form a system, and this system can, therefore, be described by using Equation (5). The equilibrium constant of the reaction can be expressed as (for more details, see Appendix B, Equation (A24)):

$$
K_{c}=\frac{z_{B}}{z_{A}} e^{-\frac{\Delta E_{0}}{R T}}
$$

where $z_{B}$ and $z_{A}$ are the partition function for $A$ and $B$, respectively and $\Delta E_{0}$ is the separation of zero-point energies. In the conversion of molecular energy to molar energy, the constant $k_{b}$ is further substituted by the constant $R$ in light of the following relation $k_{b}=R N_{A v}$ where $N_{A v}$ is the Avogadro number.

This expression serves as a link between Boltzmann's work (Equation (5)) and the transition state theory (TST). More details and explanations about this development are given in Appendices A and B and in the following references [27,30].

The basis of kinetic theory can be found in Wilhelmy, Guldberg and Waage [11], Van't Hoff [19] and Arrhenius [10]. In parallel to the kinetic theory, developments in statistical physics led to the transition state theory (TST) $[11,25,31]$ and the activated complex theory $[25,32,33]$. Using transition state theory (TST) as described in [32], the following well-known expression can be established (see Appendix B for details):

$$
k=A^{p f} e^{-\frac{E_{a c t}}{R T}}
$$

In this equation, $k$ is the kinetic equation (Arrhenius form), $A^{p f}$ is the pre-exponential factor and $E_{\text {act }}$ is the activation energy.

For Williams [34], the TST approach enables the possibility of describing a greater variety of reactions, including those due to the vibrational mode. Nevertheless, this remains in the context of homogeneous gas-phase reactions consistent with the statistical formulations. The question is now how to interpret the pre-exponential factor (and the partition function, which is part of this factor, (see Appendix B)) for different types of reactions, in particular those occurring in a solid (ex. reference [35]).

In gas-phase theory, collision theory was developed in 1916 by Trautz and in 1918 by Lewis $[32,36,37]$ and allows interpreting the kinetic law and its parameters. Subsequently, developing TST allowed Laidler and Glasstone (from 1935) to apply these concepts to a large diversity of reactions [25,38], such as:

- Elementary reactions in solutions; 
- Surface reactions, adsorption, chemisorption, etc.;

- Gas-phase reactions, single-molecule or multi-molecule.

In all of these cases, each time, these authors found a different form for the preexponential factor, but the general expression of Equation (7) remained. Given that the differences can be described through the pre-exponential factor, it is possible to infer that for conventional (chemical reaction) and unconventional applications, the Arrhenius expression is applicable so long the differences are confined to the pre-exponential factor. For this to be the case, the reaction needs to possess an energy barrier. Laidler's work $[25,38]$ well described the transition from a stable state to an activated state. This transition needs a supply of energy called the energy barrier $[25,38]$. Thus, on this basis, Brennan et al. argue the universality of the Arrhenius law [39]. Further, studies have shown that it is possible to successfully fit a form of the Arrhenius equation to represent the kinetics of any reactions that possess an energy barrier [40]. It can, therefore, be concluded that it is possible to use these representations of a reaction rate for the reactions involved in the thermal decomposition of a solid despite the absence of a clear theoretical basis.

In solid matter, all the atoms vibrate but stay in fixed, meaning positions because of their strong attractions. Consequently, we assume that the translation and rotation motions do not exist, and their respective partition functions are zeroes. Furthermore, the electrical partition function is also considered negligible because its contribution is considered too low in front of the excited state of the atoms or molecules at an ordinary temperature [38]. Then, the degradation of molecules is only due to the bond breaks. The bond breaks are the consequence of the high level of vibrational energy.

Finally, for solid reactions, the problem is reduced to vibration inside a solid matrix. This reduction conserves the Arrhenius form Equation (7) and changes the interpretation of the pre-exponential factor, which is only now linked to a vibrational mode. Furthermore, studies by the Eyring [33], Polanyi and Wigner [41] and the interpretation provided by Young [42] indicate that Equation (7) is an appropriate representation of the relevant thermodynamic principles described by Eyring [33] when only the vibrational mode is considered.

In Equation (7), the activation energy depends on temperature. To simplify the equation, Ellingham's approximation is frequently used. This approximation indicates that the condition that enables the approximation of $E_{a c t}$ to a constant is a negligible standard heat capacity for the reaction.

Through using Equations (6) and (7) (and the detailed development in Appendix B), it has been shown that the Arrhenius structure can be developed from the Boltzmann distribution for a solid. Nevertheless, the key assumptions that need to be validated when applying this approach include demonstrating a negligible standard heat capacity and the presence of an energy barrier that allows for all the differences to be included within the pre-exponential factor.

\section{Applying the Kinetic Model to a Solid Reaction}

Often, a good agreement between experimental results and models serves as the only justification for the adequacy of a modeling approach (for example, [43-47]). The adequacy of the results can many times be attributed to the way the experimental data are fitted to the model to extract the relevant constants. Indeed, the kinetic models are optimized with data obtained at a scale free of transport processes ( $\approx$ mg typically TGA scale) and using inverse methods, which can only partially guarantee the good results obtained (for more details about inverse modeling, see $[15,48])$. In the case of fire models, data fits used to optimize kinetic models will be used under conditions that drastically differ from those of instruments, such as a TGA. The extrapolation, therefore, required extreme rigor and a solid theoretical basis.

If a degradation model is necessary to predict fire behavior, and such a model is to be used under conditions that are very different from the experiments used to justify its 
validity, then using any mathematical formulation for thermal decomposition needs to be adequately justified.

The application of kinetic theory (see Section 3) in the domain of solid reactions has been the subject of criticism after the 1950s. For example, Paul Garn, through several studies published between 1965 and 1990 about reactions in crystal solids [12,49-54], indicates alternative means of representing decomposition rates. Garn's main criticism focuses on using the Boltzmann distribution to justify the Arrhenius law for a solid reaction and is argued on the demonstration that this approach leads to a large variety of parameter values found for identical reactions in the literature.

This discrepancy about parameters is partially due to the compensation effect (for details about the compensation effect, see [55]). Several authors have worked to reduce this source of uncertainties. Furthermore, the ICATC project in 2000 [56-60] aims to answer the discrepancy problem by comparing the mathematical methods used with a simple one-step mechanism. After a review of the ICTAC kinetics project in 2009, Dickinson and Heal $[13,14]$ conclude about the mathematical functions used (based on the Arrhenius form): "The process is the same as saying that a polynomial equation can be fitted exactly to any data provided a sufficient number of terms is included, and there is not a theoretical meaning to the results." These authors reach this conclusion because the Arrhenius parameters (see Equation (7) parameters $A^{p f}$ and $E_{a c t}$ ) cannot be found without an optimization process (inverse modeling). Then, these unknown parameters allow a degree of liberty to optimize the model and fit the experimental data well. The recent works of the ICTAC committee [61] do not change these conclusions. Consequently, Garn's main criticism remains, and it is not possible to justify the Arrhenius equation with experimental data.

Section 3 shows the importance of the Boltzmann distribution to establish a kinetic law. As postulated in Equation (3), the Boltzmann distribution emerges when no strong interactions between particles can be assumed. By definition, in solids, strong interactions exist. This represents the main fundamental criticism of the Arrhenius form.

Several authors have addressed the issues related to the assumptions embedded in the Boltzmann distribution by using different statistical distributions, for example, by Galwey and Brown [17]. Some of them have serious doubts about using statistical physics for solid kinetics through the activated approach and want to backtrack to the original work of Van't Hoff through a thermochemical approach $[62,63]$. However, both approaches have different interpretations of the kinetic parameters, but they are both based upon the Boltzmann statistical theory of energy distribution. Furthermore, Michel $[64,65]$ connects the statistical theory for kinetics to thermodynamics through a probabilistic rate theory and solves what the author calls the "inconsistencies of the theories".

For an overview of the works done in the past 150 years, see $[11,13,17,38,62,63,66]$. Attempts to justify the Arrhenius equation for solid kinetic seem to have reached a dead-end, and no one seemed to be able to provide any further evidence to quantify the impact of the interactions between particles on the validity of Equation (3) and the subsequent Boltzmann distribution. In contrast, the evidence of a significant influence of particle interactions was clearly justified by Garn's [12] observations on the quantitative discrepancies of the literature values used to represent the kinetic constants for solids. As a result, the common approach to applying the Arrhenius formulation for solids has been its adaptation using additional dependencies. Since the 1950s, several such adaptations of the Arrhenius law for solid applications have appeared in the literature. Examples of such adaptations are the isoconversional method, the inclusion of a temperature dependency on the pre-exponential factor and the activation energy, etc. Nevertheless, all these studies use the Arrhenius form developed using the Boltzmann statistical distribution. Therefore, they are just empirical interpretations that do not address the key issues explained above.

\section{Black Body Radiation to Answer the Problem}

A theoretical response to the applicability of the Boltzmann distribution in the Arrhenius relation for the solid degradation based on solid-phase kinetics is clearly not 
forthcoming. Consequently comes the necessity to investigate the problem from a different perspective.

Reactions within a solid are analogous to radiation in that the vibration of magnetic dipoles (atoms) and hence radiation also depends on the vibrational state of the matter. Planck [67] postulates a law that enables the representation of radiative heat transfer from a perfect solid (blackbody radiation) $[68,69]$. Planck starts by representing the spectral energy density of radiation by:

$$
U(v, T)=A^{c t} \bar{E}
$$

Here $U(v, T)$ represents the spectral energy density as a function of the frequency of the harmonic oscillator $(v)$ and the temperature $(T), A^{c t}$ is a constant and $\bar{E}$ is the average energy of the system. In this equation, the quantity $A^{c t}$ and $\bar{E}$ must be found. The complete development of Planck's law is detailed in reference [68].

To find the average energy $\bar{E}$, we have first to describe the repartition of the particles through different energy levels (see [68]). This description needs micro-statistics and applying Boltzmann statistics, see Appendix A Equation (A17), leading to the following expression:

$$
\varepsilon_{j}=E_{j} n_{j}=E_{j} \frac{N}{Z} e^{-\frac{E_{j}}{k_{b} T}}
$$

Here, $\varepsilon_{j}$ represents the total energy for the energy state $j$. Considering that, $U=\sum_{j} \varepsilon_{j}=$ $\sum_{j} E_{j} n_{j}$ (see Equation (3)) and that Equation (9) is also used in the kinetic law development (see Equation (5), Appendix A Equation (A17), Appendix B Equation (A22)) it can be stated that the kinetic model and radiative transfer model are both based on the Boltzmann distribution (see Appendix A and [68]). Here, we have a convergence between the kinetic model and the radiative transfer model.

Finally, according to reference [68], Planck gives the following expression:

$$
B^{p}(\lambda, T)=-\frac{2 h c^{2}}{\lambda^{5}\left(e^{\frac{h c}{\lambda k_{b} T}}-1\right)}
$$

where $B^{p}$ is the spectral radiance of a body at absolute temperature $T, \lambda$ represents the wavelength, $c$ the speed of light, $h$ the Planck constant and $k_{b}$ the Boltzmann constant. Equation (10) is called Planck's law and is largely used for heat transfer by radiation. Furthermore, integrating Planck's law leads to the Stefan-Boltzmann law (energy $Q=\sigma T^{4}$ with $\sigma$ the Stephan-Boltzmann constant).

An interesting point in relation to Equation (10) is it uses the same theoretical basis as the Arrhenius equation (see Appendix B). Therefore, by analogy, the radiative approach allows using Equation (10) to argue the validity of the Boltzmann distribution for solid degradation. Furthermore, Equation (10) is not constituted by unknown parameters or constants that require optimization against experimental data. Consequently, with Planck's law, the limitations about the Boltzmann distribution use are no longer appropriate.

The previous development made by Planck is accurate for a blackbody, but its application to real materials requires the implementation of some approximations. Indeed, Equation (10) is based on the Boltzmann statistics, such as the Arrhenius equation, then all components of the system (atoms) are considered identic and distinguishable (see Section 3.1). However, in real materials, all the atoms and the links between these atoms are different. Consequently, some degrees of vibrations (wavelength) are more likely than others. Therefore, it is necessary to contrast a real material to a close to perfect material. Figure 1 presents the difference between real materials (experimental curve, obtained with a multispectral camera and a wood sample with a black painted surface) and perfect material (curve calculated by Planck's law for a gray body [70], $\varepsilon=0.95$ ). Figure 1 illustrates the classical representation of the difference between the gray body model and the real material. 


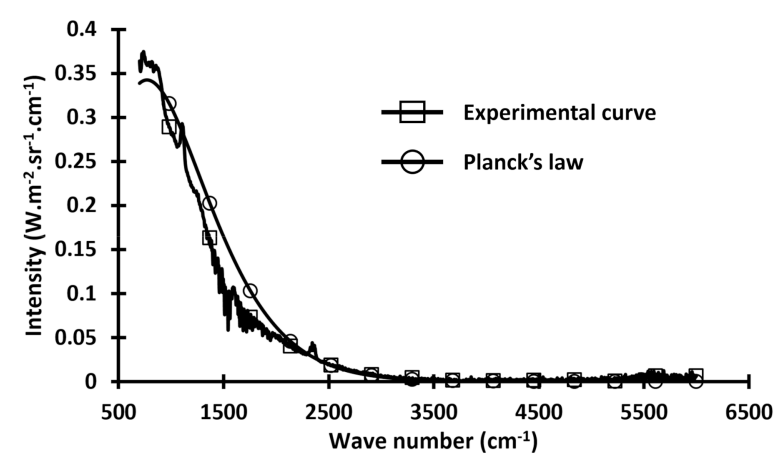

Figure 1. Spectral emission at a temperature of $393.28 \mathrm{~K}$ for a gray body and for a real body.

In this figure, the difference between the gray body and the real body highlights the differences introduced by the approximation made by using the Boltzmann distribution in Planck's law. In Figure 1, we see that the "real curve" is disturbed, but it follows the gray body model. Consequently, the Boltzmann distribution can be used to approximate the distribution of the vibrational energy through the solid. While this approximation considers all atoms or molecules identic and distinguishable, it provides a trend consistent with real measured values. In a similar manner, it is also clear that there is an error associated with the approximation. Hence, while this approach adds further justification to using the Arrhenius form, it also serves to highlight the importance of adequate means to quantify the errors.

In Equation (10), there are no unknown parameters and no compensation effect contrary to the Arrhenius equation (Equation (7)). Hence, in this section, radiative physics has been used to answer the first problem postulated by Garn [12] regarding the justification of the statistical distribution of Boltzmann statistics for solid degradation. However, the approach used is valid for elementary reactions, and this approach cannot be used when addressing the large number of elementary reactions involved during the fire-induced degradation process. It is, therefore, clear that partial justification has been achieved, but there are still significant questions left that require the attentive application of this kinetic expression.

\section{Conclusions}

In this paper, the theoretical underpinnings of the Arrhenius expression are explored, and it has been shown that this expression cannot be fully justified theoretically to be used for thermal degradation of solids, in particular in the case of fires.

The derivation follows using Boltzmann statistics to highlight the main assumptions associated with the theoretical validity of the Arrhenius expression. It was established that for mathematical development, the interactions between molecules must be considered negligible and that the particles must be identical and distinguishable. The validity of these assumptions has yet to be demonstrated for materials of the complexity of those involved in fires. Furthermore, the literature shows ample evidence of deviations from these assumptions.

The analysis of blackbody radiation can be used as an analogy that serves to further justify using the Arrhenius formulation. This approach is free of many of the empiricisms used in solid-phase kinetics; nevertheless, while demonstrating the adequacy of the trends, it also highlights the potential errors associated with using Boltzmann statistics for real materials.

Finally, in the case of fire models, data fits used to optimize kinetic models will be used under conditions that drastically differ from those of instruments, such as a TGA. Therefore, using kinetic models (Arrhenius or alternatives) and their extrapolation requires extreme rigor and a solid theoretical basis. 
Author Contributions: Investigation-Writing, T.R., F.R., J.L., A.C., E.G. and J.L.T.; InvestigationWriting-review \& editing, B.B. All authors have read and agreed to the published version of the manuscript.

Funding: This research received no external funding.

Informed Consent Statement: Not applicable.

Data Availability Statement: The data presented in this study are available on request from the corresponding author.

Acknowledgments: This work pertains to the French Government program "Investissements d'Avenir" (LABEX INTERACTIFS, reference ANR-11-LABX-0017-01). We also thank a lot the "Institut des Risques Industriels Assurantiels et Financiers" of the University of Poitiers for its technical support.

Conflicts of Interest: The authors declare no conflict of interest.

\section{Appendix A. Boltzmann Equation}

Boltzmann uses statistical mechanics $[25,28]$ to develop the path between the microscopic and macroscopic scale. To account for the micro-state, in Boltzmann's statistics, the particles must be identic and distinguishable [29]. In this case, this assumption necessary for atoms or molecules. For all the microstates [27,29]:

$$
Q \approx \frac{N !}{\prod_{i=0}^{I} n_{i} !}
$$

Here, $Q$ represents the number of configurations, $N$ is the total number of molecules and $n_{i}$ the number of molecules in the energetic state $i$. With Sterling's approximation $(\ln x ! \approx x \ln x-x)$, the solution to Equation (A1) gives (by using $\left.\sum_{i} n_{i}=N\right)$ :

$$
\ln (Q)=N \ln (N)-\sum_{i} n_{i} \ln \left(n_{i}\right)
$$

The domination configuration is when $Q$ is at a maxim, $d \ln Q=0$, then, when $Q$ is a maximum:

$$
d \ln (Q)=\sum_{i} \frac{\partial \ln (Q)}{\partial n_{i}} d n_{i}=0
$$

To solve Equation (A3), we need to use the Lagrange multiplier and the two further constraints. The first concerns energy ( $U=\sum_{i=0}^{I} E_{i} n_{i}$ see Equation (3)) as expressed by $\sum_{i} E_{i} d n_{i}=0$ and the second concerns species $\left(N=\sum_{i} n_{i}\right.$ see Equation (4)) as expressed by $\sum_{i} d n_{i}=0$.

With the Equation (A3) and the constraints, the Lagrange multiplier gives:

$$
\sum_{i} \frac{\partial \ln (Q)}{\partial n_{i}} d n_{i}+\alpha \sum_{i} d n_{i}-\beta \sum_{i} E_{i} d n_{i}=0
$$

where $\alpha$ and $\beta$ are constant. All the $d n_{i}$ are treated as independent. Then, to satisfy $d \ln Q=0$, the following equation is obtained:

$$
\frac{\partial \ln (Q)}{\partial n_{i}}+\alpha-\beta E_{i}=0
$$


The first term of this equation can be transformed by considering the Equation (A2) under the following form:

$$
\frac{\partial \ln (Q)}{\partial n_{i}}=\frac{\partial N \ln (N)}{\partial n_{i}}-\sum_{j} \frac{\partial n_{j} \ln \left(n_{j}\right)}{\partial n_{i}}
$$

In this equation, $\frac{\partial N \ln N}{\partial n_{i}}$ can be derived and gives $\frac{\partial N \ln N}{\partial n_{i}}=\ln (N)+1$ and $\sum_{j} \frac{\partial n_{j} \ln \left(n_{j}\right)}{\partial n_{i}}$ can be derived and gives to $\sum_{j} \frac{\partial n_{j} \ln \left(n_{j}\right)}{\partial n_{i}}=\ln \left(n_{i}\right)+1$. To do this derivation, the following equation is needed: $\frac{\partial N}{\partial n_{i}}=\frac{\partial \sum_{i} n_{i}}{\partial n_{i}}=1$. Then, the Equation (A6) gives:

$$
\frac{\partial \ln (Q)}{\partial n_{i}}=-\ln \left(\frac{n_{i}}{N}\right)
$$

Finally, with the Equation (A7), the Equation (A5) gives:

$$
-\ln \left(\frac{n_{i}}{N}\right)+\alpha-\beta E_{i}=0
$$

Therefore:

$$
\frac{n_{i}}{N}=e^{\alpha} e^{-\beta E_{i}}
$$

The determination of $\alpha$ gives (with Equation (A9) and $N=\sum_{i} n_{i}$ ):

$$
e^{\alpha}=\frac{1}{\sum_{i} e^{-\beta E_{i}}}
$$

With the Equations (A9) and (A10):

$$
\frac{n_{i}}{N}=\frac{e^{-\beta E_{i}}}{Z}
$$

$Z$ is the partition function.

$$
Z=\sum_{i} e^{-\beta E_{i}}
$$

To find $\beta$, the Equation (A11) is injected into the Equation (A2) to give:

$$
\ln (Q)=N \ln (N)-\sum_{i} \frac{N}{Z} e^{-\beta E_{i}} \ln \left(\frac{N}{Z} e^{-\beta E_{i}}\right)
$$

The development of the Equation (A13) gives:

$$
\ln (Q)=N \ln (N)-\sum_{i}\left(\frac{N}{Z} \beta E_{i} e^{-\beta E_{i}}+\frac{N}{Z} e^{-\beta E_{i}} \ln \frac{\mathrm{N}}{\mathrm{Z}}\right)
$$

With Equation (3), $U=\sum_{i=0}^{I} E_{i} n_{i}=\sum_{i=0}^{I} E_{i} \frac{N}{Z} e^{-\beta E_{i}}$ and Equation (4), $N=\sum_{i} n_{i}=$ $\sum_{i} \frac{N}{Z} e^{-\beta E_{i}}$. Then, the Equation (A14) gives:

$$
\ln (Q)=-\beta U+N \ln Z
$$

Which allows determining $\beta$ by using Equation (A15) ( $N \ln Z$ is a constant). The result being:

$$
\frac{\partial \ln Q}{\partial U}=-\beta
$$


With the approximation $\ln Q \approx \ln \Omega$ (with $\Omega$ the number of micro-states) and $S=$ $k_{b} \ln \Omega$, Equation (A14) gives:

$$
\frac{\partial S}{k_{b} \partial U}=-\beta
$$

$k_{b}$ is the Boltzmann constant and $S$ the entropy.

If $\frac{\partial S}{\partial U}=\frac{1}{T}$, then we obtain:

$$
\beta=-\frac{1}{k_{b} T}
$$

Equation (A12) gives:

$$
\frac{n_{i}}{N}=\frac{1}{Z} e^{-\frac{E_{i}}{k_{b} T}}
$$

Thus, Equation (A19) represents the Boltzmann distribution, which describes a number $n_{i}$ of particles in the energetic state $E_{i}$. This equation was established in 1872 by Boltzmann [29].

To simplify the previous development, degeneracy has not been considered. However, to consider the degeneracy, the same step can be used with a starting point by replacing the Equation (A1) by:

$$
Q \approx \frac{N !}{\prod_{i=0}^{I} n_{i} !} \prod_{i=0}^{I} g_{i}^{n_{i}}
$$

Finally, we obtain the Equation (A21):

$$
\frac{n_{i}}{N}=\frac{g_{i}}{Z} e^{-\frac{E_{i}}{k_{b} T}}
$$

where $g$ represents the degeneracy of the energy.

\section{Appendix B. Arrhenius Equation}

Appendix B.1. Equilibrium Constant

We consider a reaction at equilibrium in which a reactant $A$ is transformed to a product $B$ (reactants and products can be solids, liquids or gaseous). $A$ and $B$ form a system, and this system can, therefore, be described by Equation (A22) and gives:

$$
\begin{gathered}
N_{A}=\sum_{a} n_{a}=\frac{N}{Z} \sum_{a}\left(g_{a} e^{-\frac{E_{a}}{k_{b} T}}\right)=\frac{N}{Z} Z_{A} \\
N_{B}=\sum_{b} n_{b}=\frac{N}{Z} \sum_{b}\left(g_{b} e^{-\frac{E_{b}^{\prime}}{k_{b} T}}\right)=\frac{N}{Z} \sum_{b}\left(g_{b} e^{-\frac{E_{b}}{k_{b} T}}\right) e^{-\frac{\Delta E_{0}}{k_{b} T}}=\frac{N}{Z} Z_{B} e^{-\frac{\Delta E_{0}}{k_{b} T}}
\end{gathered}
$$

In the system of $A$ and $B$ molecules, $E_{b}^{\prime}=E_{b}+\Delta E_{0}$, where $\Delta E_{0}$ is the separation of zero-point energies.

In this equation, $\beta$ is replaced by $-\frac{1}{k_{b} T}$, whereas in the conversion of molecular energy to molar energy, the constant $k_{b}$ is further substituted by the constant $R$ in light of the following relation $k_{b}=R N_{A v}$ where $N_{A v}$ is the Avogadro number.

Finally, the equilibrium constant of the reaction can be expressed as:

$$
K_{c}=\frac{\mathrm{N}_{\mathrm{B}}}{\mathrm{N}_{\mathrm{A}}}=\frac{z_{B}}{z_{A}} e^{-\frac{\Delta E_{0}}{R T}}
$$

This expression serves to link Boltzmann's work (Equation (A17)) to transition state theory, which will be described in the next section. More details and explanations about this development are given in the following references [27,30]. 


\section{Appendix B.2. Application to Global Kinetic Theory}

In the case of a particle $A$ transformed to $B$. This transformation can be described through transition state theory. This description uses an intermediate species called the activated complex noted $A^{\ddagger}$. This theory indicates that particle $A$ needs to be activated before the transformation can occur. Furthermore, the transition between $A^{\ddagger}$ and $\mathrm{B}$ is considered instantaneous in front of the equilibrium formed by the transition $A$ to $A^{\ddagger}$. Then, the speed of the reaction can be written as:

$$
v=k N_{A}=k^{\ddagger} N_{A^{\ddagger}}
$$

Here, $v$ represents the reaction speed, $k$ is the reaction rate of the reaction $A$ to $B, k^{\ddagger}$ is the reaction rate for the transformation $A$ to $A^{\ddagger}$. The Equation (A25) gives:

$$
k=k^{\ddagger} K_{c}^{\ddagger}
$$

With the equilibrium constant $K_{c}^{\ddagger}=\frac{N_{A \ddagger}}{N_{A}}$.

To describe $N$ in a specific state (here $A$ or $A^{\ddagger}$ ), we can use the macroscopic scale, but we need the means to transition from the microscopic scale to the macroscopic scale. In the case of molecules, this transition is made with the Boltzmann statistical distribution (see the previous section). By using Equation (A24) to describe the equilibrium constant, we obtain the following equation:

$$
k=k^{\ddagger} \frac{Z_{A^{\ddagger}}}{Z_{A}} e^{-\beta\left(\Delta E_{0}\right)}
$$

The activated energy noted $E_{a c t}$ is $E_{a c t}=\Delta E_{0}$. Furthermore, the pre-exponential part can be represented by $A^{p f}$ and then, Equation (A27) gives:

$$
k=A^{p f} e^{-\frac{E_{a c t}}{R T}}
$$

The Equation (A28) is the same form as the Arrhenius equation experimentally determined in 1884 [20].

\section{Appendix B.3. Interpretation of the Pre-Exponential Part}

In the pre-exponential part of the Equation (A27), the partition function can be decomposed into four types of internal energy (see Equation (A29)).

$$
Z=z_{v} z_{r} z_{t} z_{e}
$$

Here $z_{v}$ is the partition function for the vibrational mode, $z_{r}$ for the rotational mode, $z_{t}$ for the translational mode and $z_{e}$ for the electronic mode. The mathematical expression of these internal energies can be found in [38]. Furthermore, Shannon [35] gives an example of how to calculate the ratio $\frac{Z_{A \ddagger}}{Z_{A}}$.

Consequently, the aims of the kinetic analysis for a reactant system is to correctly describe the partition function as a function of the matter state (liquid, solid or gas). In the kinetic theories, the gas phase has been covered before the condensed phase (liquid and solid).

Furthermore, studies by the Eyring [33], Polanyi and Wigner [41] and the interpretation provided by Young [42] indicate that Equation (7) is an appropriate representation of the relevant thermodynamic principles described by Eyring [33].

$$
k=\frac{k_{B} T}{h} \cdot e^{\frac{-\Delta S^{\ddagger}}{R}} \cdot e^{\frac{-\Delta H^{\ddagger}}{R T}}
$$

Here, $\Delta S^{\ddagger}$ represents the entropy variation during the transition and $\Delta H^{\ddagger}$ Represents the enthalpy variation during the transition. In this equation, $\Delta H^{\ddagger}$ is interpreted as the minimum energy needed for the reaction, such as the activation energy noted $E_{a c t}$. Indeed, 
the works of Eyring [33] interpret $k^{\ddagger} \frac{Z_{A \ddagger}}{Z_{A}}$ in the Equation (A27) as $v=k^{\ddagger} \frac{Z_{A \ddagger}}{Z_{A}}=\frac{k_{B} T}{h}$ where $h$ is the Planck constant [32]. Then, $v$ represents a frequency factor (the probability of a bond break by the vibrational mode), which describes vibration energy equal or up to the potential link energy.

\section{References}

1. Torero, J.L. Scaling-Up Fire. Proc. Combust. Inst. 2013, 34, 99-124. [CrossRef]

2. Drysdale, D. An Introduction to Fire Dynamics, 2nd ed.; John Wiley and Sons: Chichester, UK, 2011.

3. Quintiere, J.G. Fundamentals of Fire Phenomena; John Wiley and Sons: Chichester, UK, 2006.

4. Torero, J.L. Flaming Ignition of Solid Fuels. In SFPE Handbook of Fire Protection Engineering, 5th ed.; Hurley, M.J., Gottuk, D.T., Hall, J.R., Jr., Harada, K., Kuligowski, E.D., Puchovsky, M., Torero, J.L., Watts, J.M., Jr., Wieczorek, C.J., Eds.; Springer: New York, NY, USA, 2016; pp. 633-661.

5. Chaos, M.; Khan, M.M.; Krishnamoorthy, N.; de Ris, J.L.; Dorofeev, S.B. Evaluation of optimization schemes and determination of solid fuel properties for CFD fire models using bench-scale pyrolysis tests. Proc. Combust. Inst. 2011, 33, 2599-2606. [CrossRef]

6. Lautenberger, C.A.; Fernandez-Pello, C. Generalized pyrolysis model for combustible solids. Fire Saf. J. 2009, 44, 819-839. [CrossRef]

7. Stoliarov, S.I.; Leventon, I.T.; Lyon, R.E. Two-dimensional model of burning for pyrolysable solids. Fire Mater. 2014, 38, 391-408. [CrossRef]

8. Snegirev, A.Y.; Talalov, V.A.; Stepanov, V.V.; Harris, J.N. A new model to predict pyrolysis, ignition and burning of flammable materials in fire tests. Fire Saf. J. 2013, 59, 132-150. [CrossRef]

9. Rogaume, T. Thermal decomposition of solid fuels. Objectives, challenges and modelling. Fire Saf. J. 2019, 106, 177-188. [CrossRef]

10. Arrhenius, S. Uber die Reaktionsgeschwindigkeit bei der Inversion von Rohrzucker durch Säuren. Z. Phys. Chem. 1889, 4, 226-248. [CrossRef]

11. Eyring, H.; Eyring, E.M. Modern Chemical Kinetics; Reinhold Publishing Corporation: New York, NY, USA, 1964.

12. Garn, P.D. Thermoanalytical Methods of Investigation; Academic Press: New York, NY, USA, 1965.

13. Dickinson, C.F.; Heal, G.R. A review of the ICTAC Kinetics Project, 2000: Part 1. Isothermal results. Thermochim. Acta 2009, 494, 1-14. [CrossRef]

14. Dickinson, C.F.; Heal, G.R. A review of the ICTAC kinetics Project, 2000. Part 2. Non-Isothermal results. Thermochim. Acta 2009, 494, 15-25. [CrossRef]

15. Nyazika, T.; Jimenez, M.; Samyn, F.; Bourbigot, S. Pyrolysis modeling, sensitivity analysis, and optimization techniques for combustible materials: A review. J. Fire Sci. 2019, 37, 1-57. [CrossRef]

16. Burnham, A.K.; Braun, R.L. Global Kinetic Analysis of Complex Materials. Energy Fuels 1999, 13, 1-22. [CrossRef]

17. Galwey, A.G.; Brown, M.E. Application of the Arrhenius equation to solid state kinetics: Can this be justified? Thermochim. Acta 2002, 386, 91-98. [CrossRef]

18. Vyazovkin, S. Kinetic concepts of thermally stimulated reactions in solids: A view from a historical perspective. Int. Rev. Phys. Chem. 2000, 19, 45-60. [CrossRef]

19. Van't Hoff, M.J.H. Etude de Dynamique Chimique; Frederik Muller and Co.: Asterdam, The Netherlands, 1884.

20. Arrhenius, S. Recherche sur la Conductibilité Galvanique des Electrolytes; Ed Kongl. Boktryckeriet: Stockholm, Sweden, 1884.

21. Lewis, G.N. Autocatalytic decomposition of silver oxide. Proc. Am. Acad. Arts Sci. 1905, 40, 719-733. [CrossRef]

22. Czichos, H.; Saito, T.; Smith, L.E. Handbook of Metrology and Testing, 2nd ed.; Springer: Berlin, Germany, 2011.

23. Eyring, H.; Walter, J.; Kimball, G.E. Quantum Chemistry; John Wiley and Sons: Singapore, 1944.

24. Atkins, P.; Friedman, R. Molecular Quantum Mechanics, 4th ed.; Oxford University Press: New York, NY, USA, 2005.

25. Glasstone, S.; Laidler, K.J.; Eyring, H. The Theory of Rate Processes, 1st ed.; McGraw-Hill: New York, NY, USA, 1941.

26. Schrödinger, E. An undulatory theory of the mechanics of atoms and molecules. Phys. Rev. 1926, 28, 1049-1070. [CrossRef]

27. Atkins, P.; de Paula, J.; Keeler, J. Physical Chemistry, 11th ed.; Oxford University Press: Oxford, UK, 2018.

28. Evans, M.G.; Polanyi, M. Some applications of the transition state method to the calculation of reaction velocities, especially in solution. Trans. Faraday Soc. 1935, 31, 875-894. [CrossRef]

29. Boltzmann, L. Weitere Studien über das Wärmegleichgewicht unter Gasmolekülen. Wien. Ber. 1872, 66, $275-370$.

30. McQuarrie, D.A. Statistical Mechanics; Harper and Row: New York, NY, USA, 1973.

31. McQuarrie, D.A.; Gallogly, E.B.; Rock, P.A. Chimie Générale, 3rd ed.; De Boeck: Bruxelles, Belgium, 2012.

32. Laidler, K.J.; King, M.C. Development of transition-state theory. J. Phys. Chem. 1983, 87, 2657-2664. [CrossRef]

33. Eyring, H. The Activated Complex in Chemical Reactions. J. Chem. Phys. 1935, 3, 107-115. [CrossRef]

34. Williams, F.A. Combustion Theory, 2nd ed.; CRC Press: Boca Raton, FL, USA, 1994.

35. Shannon, R.D. Activated complex theory applied to the thermal decomposition of solids. Trans. Faraday Soc. 1964, 60, $1902-1913$. [CrossRef]

36. Trautz, M.; Wiss, Z. Photogr. Photophys. Photochem. 1906, 4, 166.

37. McCullagh Lewis, W.C. The calculation in absolute measure of velocity constant and equilibrium constant in gaseous systems. J. Chem. Soc. Trans. 1918, 113, 471-492. [CrossRef]

38. Laidler, K.J. Chemical Kinetic, 3rd ed.; Harper and Row: Manhattan, NY, USA, 1987. 
39. Brennan, J.F.; Shapiro, J.S.; Watton, E.C. Evaporation of liquids: A kinetic approach. J. Chem. Educ. 1974, 51, 276. [CrossRef]

40. Laidler, K.J. Unconventional Applications of the Arrhenius Law. J. Chem. Educ. 1972, 49, 343. [CrossRef]

41. Polanyi, M.; Wigner, E.Z. Über die Interferenz von Eigenschwingungen als Ursache von Energieschwankungen und chemischer Umsetzungen. Z. Phys. Chem. Abteiling A 1928, 139, 439-452. [CrossRef]

42. Young, D.A. Decomposition of Solids; Pergamon Press: Oxford, UK, 1966.

43. Rogaume, T.; Bustamante Valencia, L.; Guillaume, E.; Richard, F.; Luche, J.; Rein, G.; Torero, J.L. Development of the Thermal Decomposition Mechanism of Polyether Polyurethane Foam Using Both Condensed and Gas-Phase Release Data. Combust. Sci. Technol. 2011, 183, 627-644. [CrossRef]

44. Fateh, T.; Rogaume, T.; Luche, J.; Richard, F.; Jabouille, F. Kinetic and mechanism of the thermal degradation of a plywood by using thermogravimetry and Fourier-transformed infrared spectroscopy analysis in nitrogen and air atmosphere. Fire Saf. J. 2013, 58, 25-37. [CrossRef]

45. Marquis, D.; Guillaume, E.; Camillo, A.; Rogaume, T.; Richard, F. Existance and uniqueness of solutions of a differential equation system modeling the thermal decomposition of polymer materials. Combust. Flame 2013, 160, 818-829. [CrossRef]

46. Batiot, B. Etude et modélisation de la cinétique de décomposition thermique des matériaux solides. Application à la dégradation du bois en cas d'incendie. Ph.D. Thesis, Ecole National Supérieur de Mécanique et d'Aérotechnique, Poitiers, France, 2014.

47. Li, J.; Stoliarov, S.I. Measurement of kinetics and thermodynamics of the thermal degradation for non-charring polymers. Combust. Flame 2013, 160, 1287-1297. [CrossRef]

48. Purnomo, D.M.J.; Richter, F.; Bonner, M.; Vaidyanathan, R.; Rein, G. Role of optimisation method on kinetic inverse modelling of biomass pyrolysis at the microscale. Fuel 2020, 262, 116251. [CrossRef]

49. Garn, P.D. An examination of the kinetic compensation effect. J. Therm. Anal. 1975, 7, 475-478. [CrossRef]

50. Garn, P.D. The kinetic compensation effect. J. Therm. Anal. 1976, 10, 99-102. [CrossRef]

51. Garn, P.D. Kinetic parameters. Special Review. J. Therm. Anal. 1978, 13, 581-593. [CrossRef]

52. Garn, P.D. Introduction and critique of non-isothermal kinetics. Thermochem. Acta 1987, 110, 141-144. [CrossRef]

53. Garn, P.D. Kinetics of decomposition of the solid state: Is there really a dichotomy? Thermochem. Acta 1988, 135, 71-77. [CrossRef]

54. Garn, P.D. Kinetics of thermal decomposition of the solid state: II. Delimiting the homogeneous-reaction model. Thermochem. Acta 1990, 160, 135-145. [CrossRef]

55. Nikolaev, A.V.; Logvinenko, V.A.; Gorbatchev, V.M. Special features of the compensation effect in non-isothermal kinetics of solid-phase reactions. J. Therm. Anal. 1974, 6, 473-477. [CrossRef]

56. Brown, M.E.; Maciejewski, M.; Vyazovkin, S.; Nomen, R.; Sempere, J.; Burnham, A.; Opfermann, J.; Strey, R.; Anderson, H.L.; Kemmler, A.; et al. Computational aspects of kinetic analysis. Part A: The ICTAC kinetics project-data, method and results. Thermochim. Acta 2000, 355, 125-143. [CrossRef]

57. Maciejewski, M. Computational aspects of kinetic analysis. Part B: The ICTAC Kinetics Project-the decomposition kinetics of calcium carbonate revisited, or some tips on survival in the kinetic minefield. Thermochim. Acta 2000, 355, 145-154. [CrossRef]

58. Vyazovkin, S. Computational aspects of kinetic analysis. Part C: The ICTAC Kinetics Project—the light at the end of the tunnel? Thermochim. Acta 2000, 355, 155-163. [CrossRef]

59. Burnham, A.K. Computational aspects of kinetic analysis. Part D: The ICTAC Kinetics Project-multi-thermal-history modelfitting methods and their relation to isoconversional methods. Thermochim. Acta 2000, 355, 165-170. [CrossRef]

60. Roduit, B. Computational aspects of kinetic analysis. Part E: The ICTAC Kinetics Project-numerical techniques and kinetics of solid state processes. Thermochim. Acta 2000, 355, 171-180. [CrossRef]

61. Vyazovkin, S.; Burnham, A.K.; Favergeon, L.; Koga, N.; Moukhina, E.; Pérez-Maqueda, L.A.; Sbirrazzuoli, N. ICTAC Kinetics Committee recommendations for analysis of multi-step kinetics. Thermochim. Acta 2020, 689, 178597. [CrossRef]

62. L'Vov, B.V. Thermal Decomposition of Solid and Melts. New Thermochemical Approach to the Mechanism, Kinetic and Methodology; Springer: Budapest, Hungary, 2007.

63. L'Vov, B.V. Ups and downs in the theory of thermal decomposition of solids for 130 years. J. Therm. Anal. Calorim. 2017, 128, 593-600. [CrossRef]

64. Michel, D. A probabilistic rate theory connecting kinetics to thermodynamics. Physica A 2018, 503, 26-44. [CrossRef]

65. Michel, D. Test of the formal basis of Arrhenius law with heat capacities. Physica A 2018, 510, 188-199. [CrossRef]

66. Galwey, A.K. Theory of solid-state thermal decomposition reactions. Scientific stagnation or chemical catastrophe? An alternative approach appraised and advocated. J. Therm. Anal. Calorim. 2012, 109, 1625-1635. [CrossRef]

67. Planck, M. Ueber das Gesetz der Energieverteilung im Normalspectrum. Ann. Phys. 1901, 4, 553-563. [CrossRef]

68. Kramm, G.; Mölders, N. Planck's Blackbody radiation law: Presentation in different domains and determination of the related dimentional constants. J. Calcutta Math. Soc. 2009, 5, 37-61.

69. Rayleigh, L. Remarks upon the law of complete radiation. Philos. Mag. 1900, 49, 539-540. [CrossRef]

70. Modest, M.F. Radiative Heat Transfer, 3rd ed.; Elsevier: Oxford, UK, 2013. 